\title{
Fatores relacionados à fidelidade ao tratamento do glaucoma: opiniões de pacientes de um hospital universitário
}

\author{
Factors related to glaucoma treatment compliance: patients' opinions \\ from a University Hospital
}

\author{
Lucas Ravagnani Silva ${ }^{1}$ \\ Jayter Silva de Paula ${ }^{2}$ \\ Eduardo Melani Rocha ${ }^{3}$ \\ Maria de Lourdes Veronese Rodrigues ${ }^{4}$
}

\section{RESUMO}

Objetivo: Considerando que a não-adesão ao tratamento do glaucoma é um dos fatores de risco para a sua progressão, o objetivo do presente trabalho foi coletar informações sobre: 1) conhecimento e sentimentos dos pacientes sobre a doença; 2 ) a não-fidelidade ao tratamento e fatores relacionados; e 3) eventual influência da doença no estilo de vida participantes. Métodos: Foram realizadas entrevistas, utilizando questionário semiestruturado, com 50 pacientes com glaucoma primário de ângulo aberto. A análise das respostas foi feita utilizando a técnica do "Discurso do Sujeito Coletivo" (DSC) (qualitativa/quantitativa). Resultados: Trinta e oito por cento dos pacientes não se consideraram bem informados sobre o glaucoma. A porcentagem de pacientes que declararam nãofidelidade ao tratamento foi de $20 \%$. Os principais fatores relacionados foram: efeitos colaterais dos medicamentos (24\%); falta de informação sobre a doença (22\%); dificuldade de comunicação com o médico (14\%); dificuldades na administração do tratamento (14\%); falta de recursos financeiros para adquirir medicamentos (10\%); atitudes e crenças $(10 \%)$. Dezoito por cento dos participantes afirmaram que a doença alterou a qualidade de vida. Conclusão: Na população estudada, os fatores relacionados à não-adesão ao tratamento de glaucoma, abrangem desde o desconhecimento do que é a doença até atitudes, valores e crenças. Na sua maioria, podem causar a progressão da doença. A influência desses fatores pode ser diminuída com medidas educacionais, intervenções sobre comportamentos e atitudes, melhora da relação médico-paciente e suprimento de medicamentos.

Descritores: Glaucoma/quimioterapia; Atitude frente a saúde; Conhecimentos, atitudes e prática em saúde; Cooperação do paciente; Relações médico-paciente; Anti-hipertensivos/uso terapêutico; Soluções oftálmicas

\section{INTRODUÇÃO}

Glaucoma é a primeira maior causa de cegueira não curável no mundo $^{(1)}$. Em 2003, o Conselho Brasileiro de Oftalmologia estimava que no país houvesse 900 mil portadores dessa doença, e que, provavelmente, 720 mil estavam assintomáticos, ainda necessitando de diagnóstico ${ }^{(2)}$. Em níveis globais, estima-se em mais de 60 milhões o número de pessoas que estarão afetadas pelo glaucoma em 2010, sendo 8,4 milhões delas cegas ${ }^{(3)}$.

Os principais fatores de risco para a progressão do glaucoma podem ser divididos em oculares, sistêmicos e gerais. Os principais fatores considera- 
dos "gerais" são idade, etnia, história familiar e não adesão ao tratamento, ou insucesso deste tratamento ${ }^{(4-7)}$.

Dessa forma, é imprescindível a fidelidade ao tratamento. No entanto, há casos em que existem barreiras para essa fidelidade, como a falta de conhecimento sobre a doença, que propicia a não participação do paciente no tratamento ${ }^{(8-9)}$ e a falta de habilidade para instilação do colírio e/ou para manipular o frasco, associada a ausência de alguém que ajude administrar o medicamento ${ }^{(10)}$.

$\mathrm{O}$ custo do medicamento e a sua forma de aquisição também podem influenciar na não adesão do paciente ao tratamento, pelos pacientes menos favorecidos economicamente ${ }^{(11-12)}$. Além disso, a importância que uma pessoa dá à sua saúde e aos cuidados para mantê-la depende, também, de emoções, motivações, atitudes, hábitos e crenças aprendidos culturalmente ${ }^{(13)}$.

Uma vez que poucos detalhes acerca da percepção dos pacientes sobre glaucoma ou sobre seu tratamento são conhecidos em nosso meio, realizou-se o presente estudo, que teve como principais objetivos colher informações sobre: 1) conhecimento e sentimentos dos pacientes com glaucoma sobre a doença; 2) a não-fidelidade ao tratamento e se os fatores descritos na literatura ${ }^{(4-13)}$ a influenciam; e 3) eventual influência da doença na qualidade de vida participantes.

\section{MÉTODOS}

Foram entrevistados 50 pacientes, sendo 18 do sexo masculino, com idades variando de 47 a 91 anos (mediana $=71$ anos), com diagnóstico confirmado de glaucoma primário de ângulo aberto (GPAA) atendidos no Ambulatório de Oftalmologia do HCFMRP-USP.

As entrevistas foram realizadas com base em questionário semiestruturado, composto pelas seguintes perguntas abertas: 1) "Você sabe o que é o Glaucoma?"; 2) "Você compreende as informações que o médico te passa?"; 3 ) "Você faz o tratamento corretamente? Se não, por quê?" 4) "Fale sobre os seus medos e preocupações com relação a sua doença - Glaucoma"; 5) "A doença Glaucoma mudou alguma coisa no seu dia-a-dia ou na sua vida profissional?"

As respostas dos pacientes ao questionário foram analisadas com base na proposta do "Discurso do Sujeito Coletivo" ("DSC"), técnica de dupla representatividade - qualitativa e quantitativa ${ }^{(14)}$.

Assim, além de serem calculadas as porcentagens de respostas (levando em consideração o número de participantes), as opiniões dos pacientes foram categorizadas por conteúdo, para a construção do DSC dos diferentes grupos; Grupo 1: pacientes que têm conhecimentos sobre glaucoma; Grupo 2: pacientes com conhecimento parcial; Grupo 3: pacientes que não têm conhecimento; Grupo 4: pacientes que compreendem as informações do médico; Grupo 5: pacientes que compreendem parcialmente o que é glaucoma; Grupo 6: pacientes que não compreendem o que é glaucoma; Grupo 7: pacientes que aderem ao tratamento: Grupo 8: pacientes que não aderem; Grupo 9: pacientes que têm medos ou preocupações; Grupo 10: pacientes sem medos e preocupações; Grupo 11: pacientes que sofreram influência da doença na qualidade de vida e na profissão; Grupo 12: pacientes que não sofreram.

\section{RESULTADOS}

Quando interrogados sobre seus conhecimentos sobre o glaucoma, $62 \%$ dos pacientes informaram ter conhecimentos sobre a doença; $22 \%$ não sabiam o que é glaucoma e $16 \%$ tinham conhecimentos parciais sobre a doença. Alguns deles afirmaram não entender as informações do médico (6\%) e outros (8\%) apenas entendem parte das informações oferecidas.

Vinte e dois pacientes (44\%) afirmaram que não têm "fantasias e medos" em relação à doença, ou porque o tratamento está dando certo $(17 ; 34 \%$ do total) ou porque têm fé (5; $10 \%)$. Outros 2 pacientes (4\%) disseram ter sentido medo no início do tratamento, mas depois se conformaram. Dos outros 43 pacientes, 24 informaram ter medo de ficarem cegos $(48 \%$ do total) e 1 paciente ( $2 \%$ ) de sentir dor.

Quatorze pacientes (28\%) têm dificuldades para utilizar os colírios e 6 deles não têm ninguém para auxiliá-los na administração do tratamento.

Quarenta pacientes (80\%) consideraram que fazem o tratamento corretamente, apesar de 12 pacientes $(24 \%$ do total) relatarem efeitos colaterais incômodos. Os que não o fazem justificaram a não adesão por terem dificuldade de acesso aos remédios $(50 \%)$ ou dificuldade para instilar os medicamentos $(50 \%)$.

Nove $(18 \%)$ pacientes sofreram influência na qualidade de vida.

As idéias e opiniões e os sentimentos verbalizados pelos pacientes em relação ao glaucoma estão sintetizados nos seguintes DSC:

Grupo 1: pacientes que têm conhecimentos sobre glaucoma:

"O glaucoma está relacionado com a pressão do olho e é preciso controlar a pressão para não perder a visão. Pressão alta no olho pode matar o nervo do olho e deixar cego. Glaucoma não tem cura e que se eu não fizer o tratamento correto posso ficar cego".

Grupo 2: pacientes com conhecimento parcial sobre glaucoma:

"Só sei que esta doença deixa cego e que tenho que usar o remédio para controlar a minha pressão do olho para não perder a visão".

Grupo 3: pacientes que não têm conhecimento sobre glaucoma:

"Eu não sei o que é glaucoma, só sei que é uma doença ruim que dá no olho. É por isso que eu quero ficar curado logo".

Grupo 4: pacientes que compreendem as informações do médico:

"Os médicos que me atenderam são muito atenciosos, pois além de me analisar, conversaram comigo e me explicaram 
tudo. Os médicos tiram todas as minhas dúvidas e eu entendo tudo o que me falam".

Grupo 5: pacientes que compreendem parcialmente as informações do médico:

"Eles só me falaram que eu posso ficar cego e para usar os colírios corretamente para eu não perder a minha vista. Às vezes eles falam meio dificil e eu não entendo tudo".

Grupo 6: pacientes que não compreendem as informações do médico:

"Eles não me explicam nada sobre a doença ou falam muito complicado e eu não entendo".

Grupo 7: pacientes que aderem ao tratamento:

"Eu faço o tratamento corretamente e venho aos retornos porque esta doença deixa a gente cego e eu não quero não perder a minha visão. Eu valorizo muito a minha visão e mesmo quando eu não consigo ganhar os remédio, eu dou um jeito de comprar e se não consigo pingar os colírios peço ajuda".

Grupo 8: pacientes que não aderem ao tratamento:

"Eu só uso o remédio quando eu ganho e muitas vezes não tem no 'Postinho' eu não tenho dinheiro para comprar, porque é muito caro. Eu não consigo pingar sozinho e só pingo quando tenho ajuda. Outras vezes esqueço de usar os colírios ou confundo os horários e esqueço o que já usei.

Grupo 9: pacientes que têm medos ou preocupações relacionados ao glaucoma:

"Já ouvi falar que essa doença deixa a gente cega e eu não quero isso para mim. Perder a visão é muito triste e tenho muito medo disso. Não gosto de depender de outras pessoas. Também tenho medo de sentir dor".

Grupo 10: pacientes sem medos e preocupações relacionadas ao glaucoma:

Para esse item, as falas dos pacientes foram agrupadas em três discursos:

"Não estou preocupado, pois o médico e eu achamos o melhor para o tratamento, que está dando certo. Como a minha visão não piorou, estou tranquilo, pois isso quer dizer que o tratamento está correto".

"Entrego nas mãos de Deus, por que se Ele quiser eu não posso fazer nada. Mas quem tem fé em Deus não precisa temer nada. Ele sempre está comigo e me dá muita força".

"Já faz tanto tempo que tenho glaucoma que já acostumei e estou conformado".

Dos 50 pacientes entrevistados, 40 deles aderem ao tratamento do glaucoma, enquanto que 10 pacientes não o fazem corretamente.

O Discurso do Sujeito Coletivo de adesão ao tratamento do glaucoma é:

“... Eu sei que o glaucoma é uma doença que pode deixar a gente cega, pois se a pressão do olho estiver alta e não for controlada, o nervo do nosso olho vai morrendo e gente vai perdendo a visão e essa doença não tem cura. Por isso eu faço tratamento corretamente, pingo os colírios de maneira correta e quando tenho dificuldades peço ajuda para alguém da família. Quando eu não ganho os medicamentos, eu dou um jeito para comprar. Eu sempre vou aos retornos no hospital, pois lá o médico além de ele me analisar, ele é atencioso, conversa com a gente, explica de maneira clara e tira todas as minhas dúvidas. Eu tenho medo de ficar cego, pois é muito triste não poder ver mais as coisas do mundo, além do mais é muito difícil depender das outras pessoas para te ajudar, e como a doença do Glaucoma pode afetar a visão, eu tenho muito medo. Mas quando a gente faz o tratamento corretamente e observa que ele está dando certo, isso deixa tranquilo. Também com o tempo, a gente vai se conformando com a doença, $e$ aprendendo a conviver com ela e, sempre pedindo a Deus muita força e que a Sua vontade seja feita".

O Discurso do Sujeito Coletivo de não-adesão ao tratamento do glaucoma:

“... Eu não sei direito o que é o glaucoma, só sei que é uma doença que dá no olho. Os médicos não me explicam direito sobre a doença e também, eles falam difícil. Tenho dificuldades para pingar os colírios e não tenho ninguém para me ajudar. Às vezes eu esqueço de usar ou confundo os horários e misturo os colírios e então, não sei mais qual eu já usei. Também quando eu não ganho os medicamentos no 'Postinho', eu não uso, porque os colírios são caros e não tenho dinheiro para comprá-los. Eu não sei se essa doença do glaucoma deixa a gente cega, mas se deixar, eu não tenho medo, porque eu entrego tudo nas mãos de Deus e se eu ficar cego, é porque Ele quis".

\section{DISCUSSÃO}

Este estudo foi baseado em pesquisa qualitativa ${ }^{(15-16)}$, método adequado para investigar percepções e atitudes de pacientes. Este tipo de pesquisa vem sendo utilizado, amplamente em trabalhos no campo da Oftalmologia Comunitária - quer precedendo a aplicação de métodos quantitativos quer complementando informações obtidas por estes ${ }^{(17-21)}$. Algumas técnicas de interpretação dos dados obtidos, como a utilizada, Discurso do Sujeito Coletivo ${ }^{(14)}$ - têm a vantagem de acrescentar, às informações qualitativas, dados de estatística descritiva.

Os pacientes convidados a participar deste estudo foram colaborativos, possivelmente porque têm confiança no Serviço de Glaucoma que frequentam e porque apreciaram a oportunidade de interação verbal com um dos membros da equipe. $\mathrm{O}$ fato de quase $40 \%$ dos entrevistados não saberem o que é glaucoma, ou terem apenas informações parciais sobre a doença, indica a necessidade de melhorar a comunicação com os pacientes, fator indispensável em um bom relacionamento médico-paciente ${ }^{(22)}$. Uma relação médico-paciente adequada, somada a intervenções focadas em mudança de atitude e em disciplina são quesitos mais eficazes para o aumento da fidelidade ao tratamento do que o aprendizado cognitivo ${ }^{(23)}$. 
A porcentagem de pacientes $(20 \%)$ que declararam não aderir adequadamente ao tratamento é similar a encontrada em estudos realizados em outros serviços do país ${ }^{(24)}$ e está dentro dos limites relatados por pesquisadores estrangeiros ${ }^{(25-26)}$.

Da mesma forma, os fatores associados a baixa fidelidade ao tratamento - falta de informação sobre a doença; dificuldade de comunicação com o médico; dificuldades na administração do tratamento; falta de recursos financeiros para adquirir medicamentos; atitudes e crenças; e efeitos colaterais das drogas não diferem substancialmente dos descritos na literatura ${ }^{(4-7,23)}$.

No entanto, necessitava-se de avaliar as necessidades locais, para implantar adequadamente o pretendido programa de apoio para pacientes com glaucoma e seus familiares.

\section{CONCLUSÃO}

$\mathrm{Na}$ população estudada, a maior parte dos fatores relacionados à não-adesão ao tratamento de glaucoma pode ter menor importância na progressão da doença, por meio de medidas educativas, intervenções sobre comportamentos e atitudes, melhora da relação médico-paciente e suprimento de medicamentos.

\section{ABSTR ACT}

Purpose: Considering that noncompliance to glaucoma treatment is one of the risk factors associated with disease's progression, the purpose of the present study was to evaluate: 1 ) knowledge and feelings about the disease; 2) self-reported noncompliance to treatment and related factors; and 3) influence of disease in lifestyle. Methods: Interviews, with application of a semi-structured questionnaire, of 50 patients with primary open-angle glaucoma. The analysis was performed using Lefèvre's technique (Collective Subject Speech - CSS, qualitative/quantitative research). Results: Thirty-eight percent of patients did not consider themselves well informed about glaucoma. The percentage of patients that reported noncompliance was $20 \%$. The main causes of noncompliance are: side effects of antiglaucomatous drugs (24\%); lack of information about the disease (22\%); difficulty in understanding information and communication problems with the physician (14\%); difficulties in administering the eye drops (14\%); poverty, with impossibility to pay for the eye drops (10\%); patients' attitudes and beliefs (10\%). Eighteen percent of the participants informed that the disease affected their life quality. Conclusion: In the studied population, most of the problems related to noncompliance to glaucoma treatment pointed out different factors that may decrease with education about POAG, interventions on attitudes and behavior, improvement of doctor-patient relationship and anti-glaucoma drugs supply.

Keywords: Glaucoma/drug therapy; Health knowledge, attitudes, practice; Patient compliance; Doctor-patient relations; Antihypertensive agents/therapeutic use; Ophthalmic solutions

\section{REFERÊNCIAS}

1. Foster A, Gilbert C, Johnson G. Changing patterns in global blindness:19882008. Community Eye Health. 2008;21(67):37-9.

2. Mello PAA, Melo Júnior LAS. Glaucoma dos nossos dias: revendo conceitos e custos. Rev Bras Oftalmol.2003;62(9):669-77.

3. Quigley HA, Broman AT. The number of people with glaucoma worldwide in 2010 and 2020. Br J Ophthalmol 2006;90(3):262-7.

4. Allingham RR, Shields B, Damji KF, Freedman S, Moroi SE, Shafranov G. Shield's Textbook of Glaucoma. Philadelphia: Lippincott Williams \& Wilkins; 2005. p.170-96.

5. Boland MV, Quigley HA. Risk factors and open-angle glaucoma: classification and application. J Glaucoma. 2007;16(4):406-18

6. Bron A, Chaine G, Villain M, Colin J, Nordmann JP, Renard JP, Rouland JF. [Risk factors for primary open-angle glaucoma]. J Fr Ophtalmol. 2008; 31(4):435-44.

7. Leske MC. Open-angle glaucoma - an epidemiologic overview. Ophthalmic Epidemiol. 2007;14(4):166-72.

8. Cintra FA, Costa VP, Tonussi JA, Jose NK. [Evaluation of an educational program for patients with glaucoma]. Rev Saude Publica. 1998;32(2):172-7. Portuguese.

9. Costa VP. Spaeth GL, Smith M, Uddoh C, Vasconcelos JPC, Kara-José N. Patient education in glaucoma: what do patients know about glaucoma? Arq Bras Oftalmol. 2006;69(6):923-7.

10. Winfield AJ, Jessiman D, Williams A, Esakowitz L. A study of the causes of non-compliance by patients prescribed eyedrops. Br J Ophthalmol. 1990; 74(8):477-80

11. Silva LMS, Vasconcellos JP, Temporini ER, Costa VP, Kara-José N. Tratamento clínico do glaucoma em um hospital universitário: custo mensal e impacto na renda familiar. Arq Bras Oftalmol. 2002;65(3):299-303.

12. Kara-José N, Vasconcelos JP, Nastari ER Costa VP. Tratamento clínico do glaucoma em um Hospital Universitário: custo mensal e impacto na renda familiar. Arq Bras Oftalmol. 2002;65(3):299-303.

13. Friedman DS, Hahn SR, Gelb L, Tan J, Shah SN, Kim EE, et al. Doctorpatient communication, health-related beliefs, and adherence in glaucoma results from the Glaucoma Adherence and Persistency Study. Ophthalmology. 2008;115(8):1320-7.

14. Lefèvre F, Lefèvre AM. Os novos instrumentos no contexto da pesquisa qualitativa. In: Lefèvre F, Lefèvre AM, Teixeira JJV, organizadores. O discurso do sujeito coletivo: uma nova abordagem metodológica em pesquisa qualitativa. Caxias do Sul: EDUCS; 2000. p.11-35.

15. Turato ER. Tratado da metodologia da pesquisa clínico-qualitativa. São Paulo, Vozes; 2003. p.685.

16. Rocha EM, Alves M. Metodologia qualitativa. In: Bicas HEA, Rodrigues MLV, editores. Metodologia científica. Rio de Janeiro: Cultura Médica; 2008. p.337-40.

17. Kara-José N, Alves MR, Temporini ER. Características e percepções de pais de escolares portadores de problemas oculares atendidos em projeto comunitário. Rev Bras Oftalmol. 2000;59(2):99-104.

18. Nastari ER, Holzchuh N, Kara N Jr, Kara-José N. Popular beliefs regarding the treatment of senile cataract. Rev Saude Publica. 2002;36(3):343-9.

19. Gasparetto ME, Temporini ER, Carvalho KM, Kara-José N. Dificuldade visual em escolares: conhecimentos e ações de professores do ensino fundamental que atuam com alunos que apresentam visão subnormal. Arq Brás Oftalmol. 2004;67(1):65-71.

20. Oliveira DF, Arieta CE, Temporimni ER, Kara-José N. Qualidade da assistência à saúde: satisfação de pacientes em um Hospital Universitário. Arq Bras Oftalmol. 2006; 69(5):731-6.

21. Carvalho RS, Temporini AR, Kara-José N. Assessment of visual health campaign activities at school teachers perception. Arq Bras Oftalmol. 2007;70(2):239-45.

22. Hamelin N. [The physician-patient relationship in glaucoma]. J Fr Ophtalmol. 2008;31(6 Pt 2):2S39-44. French.

23. Hoevenaars JG, Schouten JS, van den Borne B, Beckers HJ, Webers CA. Will improvement of knowledge lead to improvement of compliance with glaucoma medication? Acta Ophthalmol. 2008;86(8):849-55.

24. Castro AN, Mesquita WA. [Noncompliance with drug therapy for glaucoma]. Arq Bras Oftalmol. 2008;71(2):207-14. Portuguese.

25. Schwartz GF. Compliance and persistency in glaucoma follow-up treatment. Curr Opin Ophthalmol. 2005;16(2):114-21.

26. Olthoff CM, Schouten JS, van de Borne BW, Webers CA. Noncompliance with ocular hypotensive treatment in patients with glaucoma or ocular hypertension an evidence-based review. Ophthalmology. 2005;112(6):953-61. 